\title{
Indirect searches for dark matter with the ANTARES neutrino telescope
}

\author{
Christoph Tönnis*, on behalf of the ANTARES Collab. \\ IFIC - Instituto de Física Corpuscular, \\ Universitat de València-CSIC, \\ E-46100 Valencia, Spain \\ Department of Physics, \\ Sungkyunkwan University, \\ Seobu 16419, Suwon, South Korea \\ E-mail: Christoph.toennisegmx.de
}

\begin{abstract}
One of the major purposes of the ANTARES neutrino telescope is the indirect search for dark matter. The ANTARES detector is located on the bottom of the Mediterranean Sea, $40 \mathrm{~km}$ off the southern French coast. In this talk the results of the search for dark matter signals from the Sun, the Galactic Center and the Earth core, produced with different analysis methods, will be presented. There are various advantages in indirect searches with neutrino telescopes when comparing to other experiments. The analysis for the Sun puts good limits on the spin-dependent scattering cross section between dark matter and hydrogen and the limits presented for the Galactic Center are the most stringent of all indirect detection experiments for WIMP masses above 30 $\mathrm{TeV}$.
\end{abstract}

35th International Cosmic Ray Conference - ICRC2017

10-20 July, 2017

Bexco, Busan, Korea

${ }^{*}$ Speaker. 


\section{Introduction}

The ANTARES detector, located $40 \mathrm{~km}$ off the shore of Toulon in southern France $2500 \mathrm{~m}$ below the surface of the Mediterranean sea, is a neutrino detector, which uses the sea water as its detector medium. It consists of 12, $450 \mathrm{~m}$ long, lines of electro-optical cable, each installed in the ocean floor and held up by a buoy. Every line is equipped with 25 so-called storeys, each containing 3 optical modules. An optical module is a 17-inch borosilicate sphere containing a 10inch photomultiplier tube (PMT) of the type Hamamatsu R7081-20 and the electronics to control it. This makes a total of 900 PMTs in the whole detector.

One of the main physics goals of neutrino telescopes is the indirect search for dark matter [1, 2]. The concept behind the search is that dark matter particle accumulate in massive objects either primordially or via scattering with the matter of the objects and annihilate inside of them, producing pairs of standard model particles. These particles then decay further, producing neutrinos in these secondary processes. The results of three of these searches, one for annihilations in the Sun, one for annihilations in the Earth and one for annihilations in the Milky Way, are presented in this proceeding. Further analyses for secluded dark matter [3] have been performed using ANTARES.

The currently most prevalent hypothesis on dark matter is that it is composed of weakly interacting massive particles (WIMPs) that form halos in which galaxies are embedded. As candidates for the WIMP typically supersymmetric particles are considered.

In the search for dark matter in the Milky Way the extension of the source had to be taken into account. This extension for searches looking for annihilations is expressed by the so-called J-Factor. The J-Factor is the squared dark matter density of the source integrated over the line of sight. It is calculated by

$$
\mathrm{J}(\theta)=\int_{0}^{1_{\max }} \frac{\rho_{\mathrm{DM}}^{2}\left(\sqrt{\mathrm{R}_{\mathrm{SC}}^{2}-21 \mathrm{R}_{\mathrm{SC}} \cos (\theta)+1^{2}}\right)}{\mathrm{R}_{\mathrm{SC}} \rho_{\mathrm{SC}, \mathrm{DM}}^{2}} \mathrm{dl},
$$

where $\mathrm{R}_{\mathrm{SC}}$ and $\rho_{\mathrm{SC}}$ are the scaling radius and density. The J-Factor is also needed to relate the neutrino flux $\frac{\mathrm{d} \phi_{v}}{\mathrm{dE}}$ to the thermally averaged annihilation cross-section $\langle\sigma \mathrm{v}\rangle$ :

$$
\frac{\mathrm{d} \phi_{v}}{\mathrm{dE}}=\frac{\langle\sigma \mathrm{v}\rangle}{2} \mathrm{~J}_{\Delta \Omega} \frac{\mathrm{R}_{\mathrm{SC}} \rho_{\mathrm{SC}}^{2}}{4 \pi \mathrm{m}_{\chi}^{2}} \frac{\mathrm{dN}}{\mathrm{dE}},
$$

where $\mathrm{J}_{\Delta \Omega}$ is the J-Factor integrated over the observation window $\Delta \Omega, \mathrm{m}_{\chi}$ is the WIMP mass and $\frac{d N_{v}}{d E}$ is the expected signal neutrino spectrum. For the dark matter halo profile $\rho_{\mathrm{DM}}$ the NFW profile was considered with parameters taken from [5].

The Sun, however, is sufficiently small to be considered a point-like source, so the source extension does not have to be taken into account. When limits on dark matter model parameters are calculated an equilibrium between the accumulation of dark matter in the Sun and their annihilation is assumed. This allows to convert limits and sensitivities in terms of neutrino fluxes to spin dependent and spin independent scattering cross-section limits $\left(\sigma_{S D}^{p}\right.$ and $\left.\sigma_{S I}\right)$ assuming a Maxwellian velocity distribution of the WIMPs with a root mean square velocity of $270 \mathrm{~km} \cdot \mathrm{s}^{-1}$ and a local dark matter density of $0.3 \mathrm{GeV} \cdot \mathrm{cm}^{-3}$ [7]. 
For the Earth no equilibrium can be asserted and instead a value for the thermally averaged annihilation cross section in the Earth, $\left\langle\sigma_{\mathrm{A}} \mathrm{v}\right\rangle_{\text {Earth }}$, is assumed.

To remain independent of particle dark matter models, limits are calculated using so-called annihilation channels. An annihilation channel is the assumption that all WIMP annihilations initially lead to the same pair of standard model particles. There are five channels that were considered:

$$
\mathrm{DM}+\mathrm{DM} \rightarrow \mathrm{b} \overline{\mathrm{b}}, \mathrm{W}^{+} \mathrm{W}^{-}, \tau^{+} \tau^{-}, \mu^{+} \mu^{-}, v \bar{v}
$$

The $v \bar{v}$ and $\mu^{+} \mu^{-}$channel have not been considered for the search for WIMP annihilations in the Sun and the Earth to simplify the analysis. The $\tau^{+} \tau^{-}$channel is most commonly used as a benchmark for comparisons between experiments. When the expected neutrino signal spectra are calculated for the Sun, the absorption of neutrinos in the solar plasma has to be taken into account. In both cases neutrino oscillations were accounted for in the calculations, which were carried out using the WIMPSIM code [8] for the Sun and using the code described in [9] for the Milky Way.

The sensitivities and limits are then converted to neutrino fluxes using a quantity referred to as acceptance. The acceptance is defined as:

$$
\operatorname{Acc}\left(m_{\text {WIMP }}, C h\right)=\left.\int_{E_{\text {th }}}^{m_{\text {IIMP }}} A_{\text {eff }}\left(E_{v_{\mu}}\right) \frac{\mathrm{dN}_{v_{\mu}}}{\mathrm{dE}_{v_{\mu}}}\right|_{\text {Det,Ch }} \mathrm{dE}_{v_{\mu}}+\left.\int_{\mathrm{E}_{\mathrm{th}}}^{\mathrm{m}_{\mathrm{WIMP}}} \mathrm{A}_{\mathrm{eff}}\left(\mathrm{E}_{\bar{v}_{\mu}}\right) \frac{\mathrm{dN}_{\bar{v}_{\mu}}}{\mathrm{dE}_{\bar{v}_{\mu}}}\right|_{\text {Det,Ch }} \mathrm{dE}_{\bar{v}_{\mu}},
$$

where $A_{\text {eff }}\left(E_{v_{\mu}}\right)$ is the effective area for the muon neutrino energy $E_{v_{\mu}}$ or muon antineutrino energy $E_{v_{\bar{\mu}}},\left.\frac{\mathrm{dN}_{v_{\mu}}}{\mathrm{dE}_{v}}\right|_{\text {Det,Ch }}$ is the signal neutrino spectrum at the position of the detector for one particular annihilation channel $\mathrm{Ch}$ listed in equation $1.3, \mathrm{E}_{\text {th }}$ is the energy threshold of the detector and $\mathrm{m}_{\text {WIMP }}$ is the WIMP mass. The effective area, which is the size of a $100 \%$-efficient detector giving the same number of events, is calculated from the Monte Carlo simulation. The $90 \%$ C.L. limits and sensitivities on the fluxes are then calculated by:

$$
\bar{\Phi}_{v_{\mu}+\bar{v}_{\mu}, 90 \%}=\frac{\bar{\mu}_{v_{\mu}+\bar{v}_{\mu}, 90 \%}\left(\mathrm{~m}_{\mathrm{WIMP}}\right)}{\operatorname{Acc}\left(\mathrm{m}_{\mathrm{WIMP}}\right) \cdot \mathrm{T}_{\text {live }}},
$$

where $\bar{\mu}_{v_{\mu}+\bar{v}_{\mu}, 90 \%}$ is the $90 \%$ C.1. sensitivity or limit and $\mathrm{T}_{\text {live }}$ is the total live time of the detector.

For the analyses presented here a maximum likelihood algorithm was used. This algorithm searches for signals using a likelihood function. For this analysis the likelihood has the form:

$$
\log _{10}\left(\mathrm{~L}\left(\mathrm{n}_{\mathrm{s}}\right)\right)=\sum_{\mathrm{i}=1}^{\mathrm{N}_{\text {tot }}} \log _{10}\left(\mathrm{n}_{\mathrm{s}} \mathrm{S}\left(\psi_{\mathrm{i}}, \mathrm{p}_{\mathrm{i}}, \mathrm{q}_{\mathrm{i}}\right)+\mathrm{N}_{\text {tot }} \mathrm{B}\left(\psi_{\mathrm{i}}, \mathrm{p}_{\mathrm{i}}, \mathrm{q}_{\mathrm{i}}\right)\right)-\mathrm{n}_{\mathrm{s}}-\mathrm{N}_{\text {tot }} .
$$

$\mathrm{N}_{\text {tot }}$ is the total number of reconstructed events, $n_{s}$ is the supposed number of signal events, $\psi_{\mathrm{i}}$ is the angular position of the $\mathrm{i}_{\text {th }}$ event, $\mathrm{p}_{\mathrm{i}}$ and $q_{i}$ are additional event parameters like the reconstruction quality or the estimated neutrino energy. $\mathrm{S}$ represents the ANTARES point spread function (PSF) and $B$ is a function that represents the behaviour of the background.

Pseudo experiments are then used to study the behaviour of the likelihood function. A pseudo experiment is a simulated event distribution, generated from a background estimate including a given number of fake signal events. For each pseudo experiment the likelihood function is optimised with regards to $\mathrm{n}_{\mathrm{s}}$ and a parameter called test statistic is calculated: 


$$
\mathrm{TS}=\log _{10}\left(\frac{\mathrm{L}\left(\mathrm{n}_{\mathrm{S}}\right)}{\mathrm{L}(0)}\right)
$$

The sensitivities in terms of detected signal events $\mu_{90 \%}$ are calculated from the overlap of the distribution of TS values for different numbers of inserted fake signal events. Upper limits on the number of signal events are then calculated comparing the TS value of the actual data to the TS distributions of pseudo experiments.

\section{Search in the Earth}

For the search for Dark matter annihilations in the Earth data recorded from 2007 to 2012 have been used. Upper limits at 90\% C.L. on the WIMP annihilation rate in the Earth and the spin independent scattering cross-section of WIMPs to nucleons were calculated for three annihilation channels. A comparison of these limits to the results of other experiments is presented in Figure 1. For masses of the WIMP close to the mass of iron nuclei $\left(50 \mathrm{GeV} / \mathrm{c}^{2}\right)$, the obtained limits are more stringent than those obtained by other indirect searches.

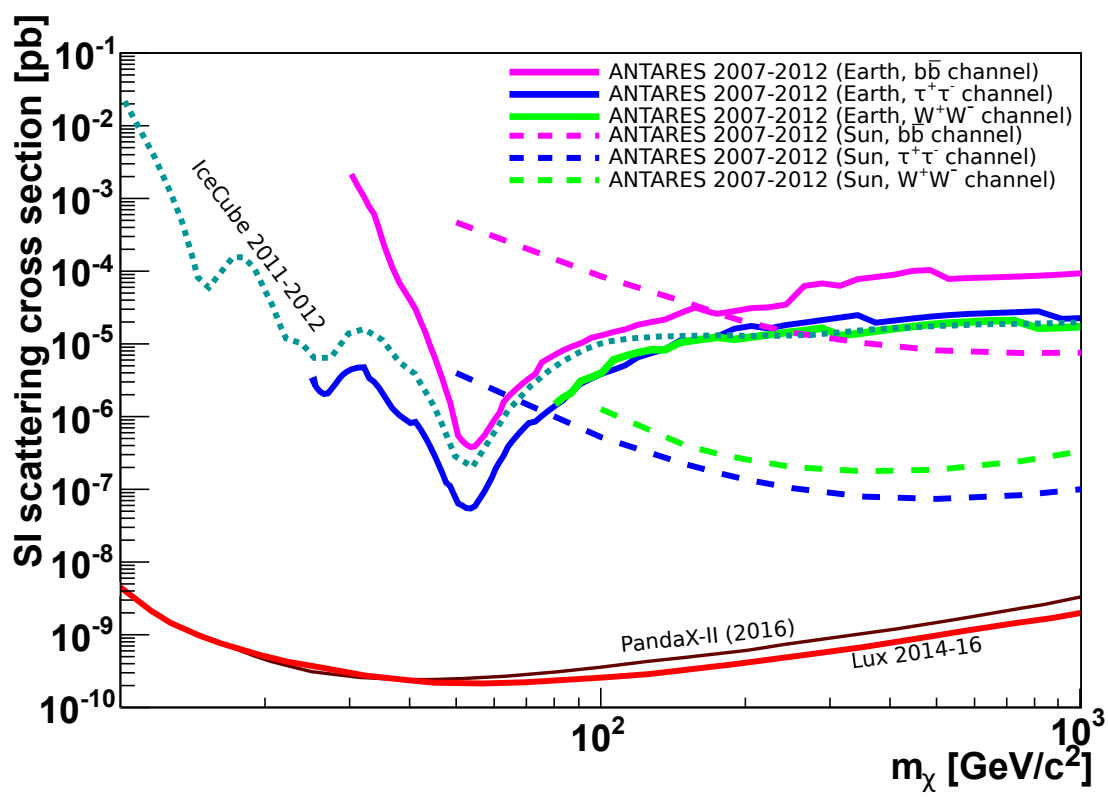

Figure 1: Limits on the spin-independent WIMP-nucleon scattering cross-section as a function of WIMP mass assuming $\left\langle\sigma_{\mathrm{A}} \mathrm{v}\right\rangle_{\mathrm{Earth}}=3 \cdot 10^{-26} \mathrm{~cm}^{3} \mathrm{~s}^{-1}$. Results from IceCube-79 [10], PandaX-II [11] and LUX [12] are shown as well.

\section{Search in the Sun}

The indirect search for dark matter in the Sun was performed using a dataset that was collected between 2007 and 2012. In that dataset no significant excess was found and therefore limits on the spin dependent and spin independent scattering cross-section were set. In Figure 2 the limits on the spin dependent scattering cross-section are compared to the results from other experiments. At low 
WIMP masses the SuperKamiokande experiment provides the most stringent limits intersecting with the limits from neutrino telescopes at $100 \mathrm{GeV}$. Above that the IceCube experiment generates the lowest limits. However the comparison to the ANTARES limits is extremely close considering the enormous difference in size between the experiments. This is possible because of the much better angular resolution of ANTARES due to the smaller amount of scattering in water compared to ice.

Since direct detection experiments are not designed to be sensitive to $\sigma_{S D}^{p}$ they can usually not compete with neutrino experiments for this parameter. Only the bubble chamber experiment PICO can provide limits in a similar order of magnitude because it uses a target material with a high density of unpaired spin.

In figure 3 the spin independent scattering cross section limit is shown in comparison to other experiments. The spin independent scattering is dependent on the abundance of helium in the Sun, whilst the spin dependent scattering depends on the abundance of hydrogen. Since helium is much less prevalent than hydrogen in the Sun the spin independent cross-section limits are much less stringent than those shown in figure 2. Direct detection experiments, being designed for a high sensitivity to spin independent scattering, provide much lower limits in comparison to those from neutrino experiments.

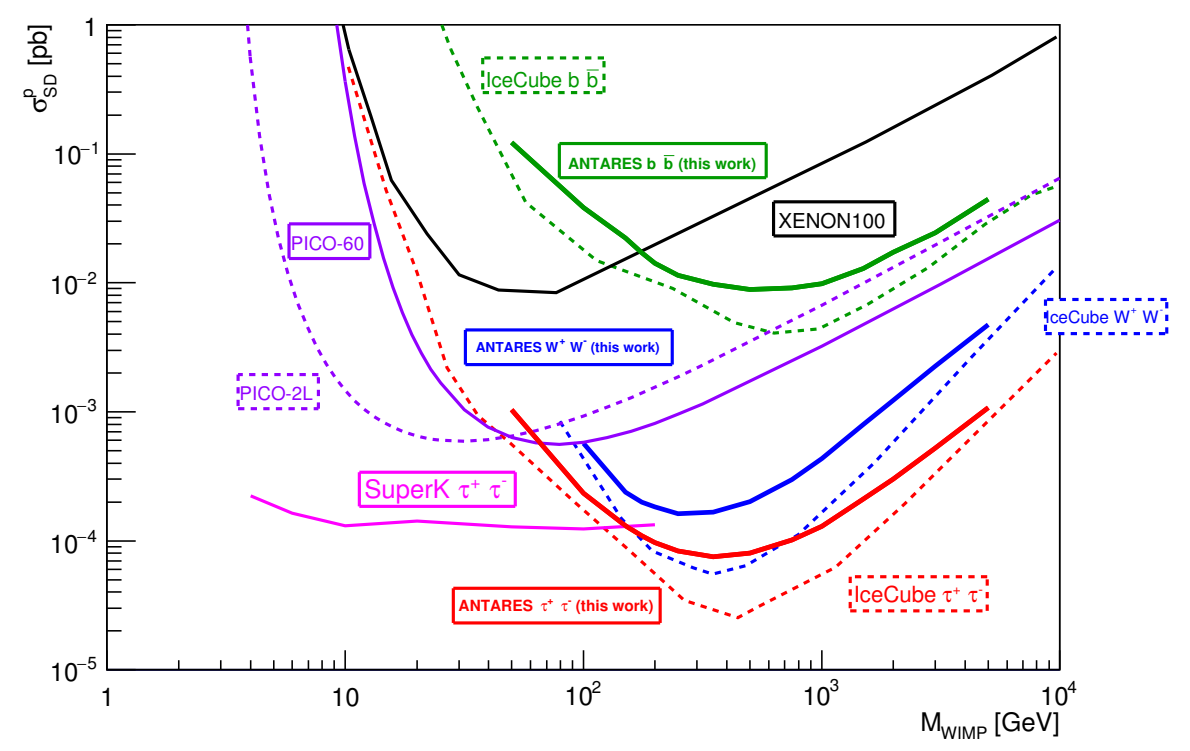

Figure 2: Limits on the spin-dependent WIMP-nucleon scattering cross-section as a function of WIMP mass for the $b \bar{b}, \tau^{+} \tau^{-}$and $W^{+} W^{-}$channels. Limits given by other experiments are also shown: IceCube [13], PICO-60 [15], PICO-2L [16], SuperK [17], XENON100 [18].

The comparison to IceCube in figure 3 is much more favourable since the limits shown there did only use muon tracks and a smaller data sample as the most recent IceCube publication for this type of search [13], which did not include limits on the spin independent cross-section. 


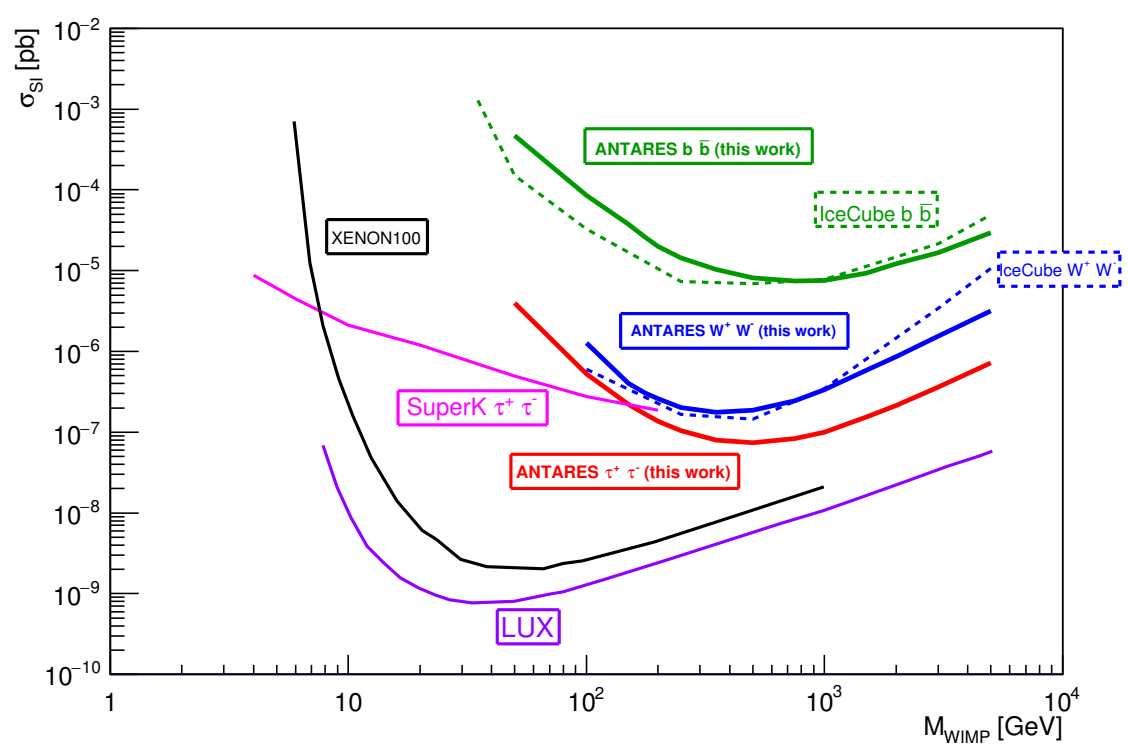

Figure 3: Limits on the spin-independent WIMP-nucleon scattering cross-section as a function of WIMP mass for the different channels considered. Limits given by other experiments are also shown: IceCube [14], SuperK [17], LUX [19], XENON100 [20].

\section{Search in the Galactic Centre}

For the search in the Galactic Centre a larger dataset, containing events from 2007 to 2015, has been used. Just as in the case of the Sun no significant excess above the expected background has been found and limits on the thermally averaged annihilation cross-section were set.

The limits on $\langle\sigma \mathrm{v}\rangle$ are compared to those from other experiments in figure 4. For all WIMP masses above $100 \mathrm{GeV}$ the limits from ANTARES supersede those from IceCube by more than one order of magnitude. This comparison is favourable since ANTARES has a good visibility towards the Galactic Centre, whilst IceCube needs to use a veto to exclude atmospheric muons. This veto reduces the effective area of IceCube, particularly at high energies.

Gamma ray experiments provide the most stringent limits for WIMP masses lower than 30 $\mathrm{TeV}$. Above that the limits from ANTARES are the most stringent. This has to be considered with the caveat that the limits from HESS use the Einasto halo profile, which is less cuspy than the NFW profile used for the ANTARES limit.

\section{Conclusions}

Despite the much smaller size of ANTARES in comparison to similar experiments competitive limits for the spin dependent scattering cross-section could be provided. Especially positive were the very strong constrains that were set on the thermally averaged annihilation cross-section, that are the currently best limits of all neutrino telescopes and even supersede limits from gamma ray experiments. Further analyses for the Earth and secluded dark matter have been performed as well $[3,4]$. 


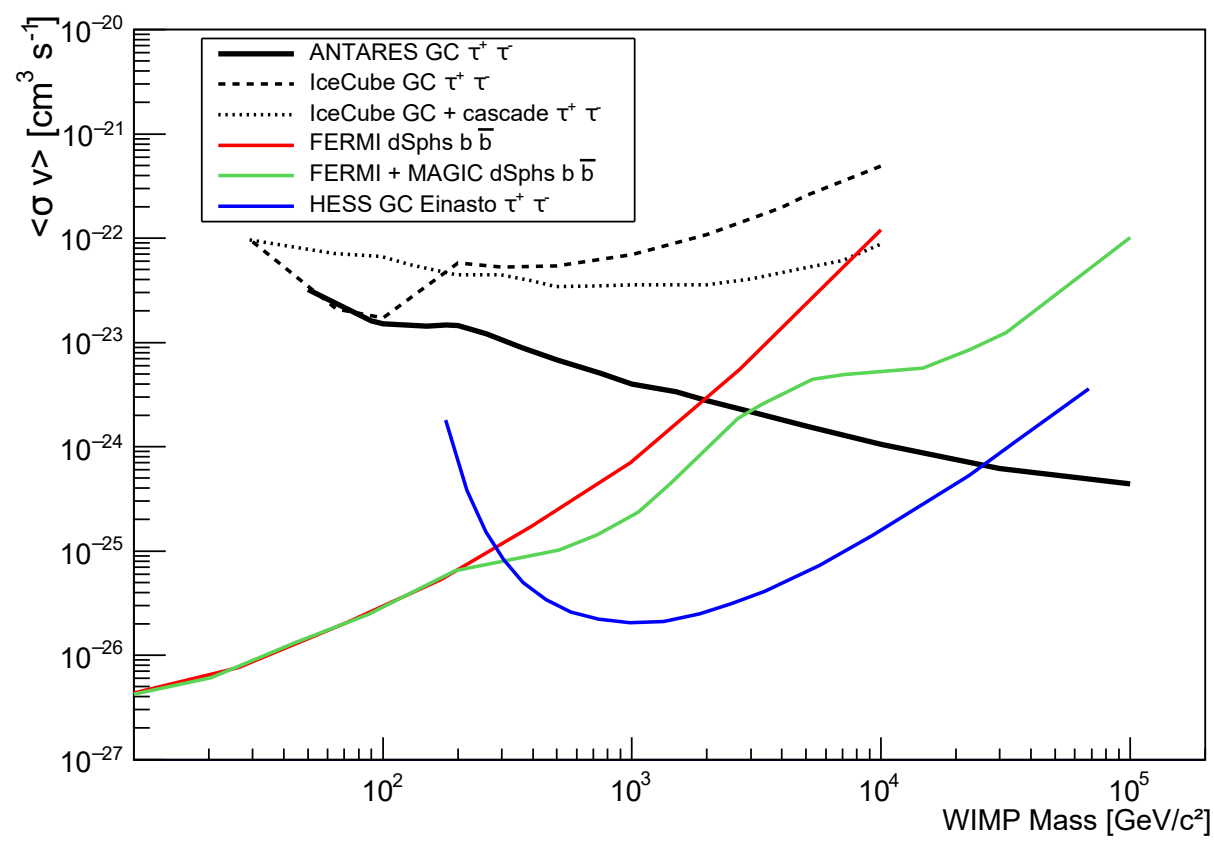

Figure 4: $90 \%$ C.L. limits on the thermally averaged annihilation cross-section, $\langle\sigma v\rangle$, as a function of the WIMP mass in comparison to the limits from other experiments [21, 22, 23, 24, 25]. The results from IceCube and ANTARES were obtained with the NFW profile.

\section{References}

[1] M. Klasen, M. Pohl, G. Sigl, Indirect and direct search for dark matter, Progress in Particle and Nuclear Physics 851 (2015)

[2] G. Bertone, D. Hooper, J. Silk, Particle Dark Matter: Evidence, Candidates and Constraints, Phys. Rep. 405279 (2005)

[3] ANTARES Collaboration, S. Adrian-Martinez et al., A search for Secluded Dark Matter in the Sun with the ANTARES neutrino telescope, JCAP 05016 (2016)

[4] ANTARES Collaboration, A. Albert et al., Search for dark matter annihilation in the earth using the ANTARES neutrino telescope, Phys. of the Dark Univ. 1641 (2017)

[5] F. Nesti, P. Salucci, The Dark Matter Halo of the Milky Way, AD 2013,JCAP 07016 (2013)

[6] Paul J. McMillan,The mass distribution and gravitational potential of the Milky Way, MNRAS 414 2446 (2015)

[7] G. Wikström, J. Edsjö, Limits on the WIMP-nucleon scattering cross-section from neutrino telescopes, JCAP 0904009 (2009)

[8] M. Blennow, J. Edsjo, T. Ohlsson, Neutrinos from WIMP Annihilations obtained using a Full Three-Flavor Monte Carlo Approach, JCAP 0801021 (2008)

[9] M. Cirelli et al., PPPC 4 DM ID: A Poor Particle Physicist Cookbook for Dark Matter Indirect Detection, JCAP 1103051 (2011) 
[10] IceCube Collaboration, M. G. Aartsen et al., First search for dark matter annihilations in the Earth with the IceCube Detector, EPJ C77 82 (2017)

[11] PandaX-II Collaboration, Andi Tan et al., Dark Matter Results from First 98.7 Days of Data from the PandaX-II Experiment, Phys. Rev. Lett. 117121303 (2016)

[12] LUX Collaboration, D. S. Akerib et al,. Results from a search for dark matter in the complete LUX exposure, Phys. Rev. Lett. 118021303 (2017)

[13] IceCube Collaboration, M. G. Aartsen et al., Improved limits on dark matter annihilation in the Sun with the 79-string IceCube detector and implications for supersymmetry, JCAP 1604022 (2016)

[14] IceCube Collaboration, M. G. Aartsen et al., Search for dark matter annihilations in the Sun with the 79-string IceCube detector, Phys. Rev. Lett., 110131302 (2013)

[15] PICO Collaboration, C. Amole et al., Dark Matter Search Results from the PICO-60 CF3I Bubble Chamber, Phys. Rev. D 93052014 (2016)

[16] PICO Collaboration, C. Amole et al., Improved Dark Matter Search Results from PICO-2L Run-2, Phys. Rev. D 93061101 (2016)

[17] Super-Kamiokande Collaboration, K. Choi et al., Search for Neutrinos from Annihilation of Captured Low-Mass Dark Matter Particles in the Sun by Super-Kamiokande, Phys. Rev. PRL 114141301 (2015)

[18] XENON100 Collaboration, E. Aprile et al., Limits on spin-dependent WIMP-nucleon cross sections from 225 live days of XENON100 data, Phys. Rev. Lett. 111021301 (2013)

[19] LUX Collaboration, D. S. Akerib et al., First results from the LUX dark matter experiment at the Sanford Underground Research Facility, Phys. Rev. Lett. 112091303 (2014)

[20] XENON100 Collaboration, E. Aprile et al., Dark Matter Search Results from 225 Live Days of XENON100 Data, Phys. Rev. Lett. 109181301 (2012)

[21] M. G. Aartsen et al., IceCube Collaboration, Search for Dark Matter Annihilation in the Galactic Center with IceCube-79, EPJC 75492 (2015)

[22] M. G. Aartsen et al., IceCube Collaboration,All-flavour Search for Neutrinos from Dark Matter Annihilations in the Milky Way with IceCube/DeepCore, EPJC 76531 (2016)

[23] M. Ackermann et al., Fermi-LAT Collaboration, Searching for Dark Matter Annihilation from Milky Way Dwarf Spheroidal Galaxies with Six Years of Fermi Large Area Telescope Data, Phys. Rev. Lett. 115231301 (2015)

[24] R. Caputo, M. R. Buckley, P. Martin, E. Charles, A. M. Brooks, A. Drlica-Wagner, J. M. Gaskins, M. Wood, Search for Gamma-ray Emission from Dark Matter Annihilation in the Small Magellanic Cloud with the Fermi Large Area Telescope, Phys. Rev. D 936062004 (2016)

[25] H. Abdallah et al., HESS Collaboration, Search for dark matter annihilations towards the inner Galactic halo from 10 years of observations with H.E.S.S, Phys. Rev. Lett. 11711111301 (2016) 